\section{Secreted factors from mouse embryonic fibroblasts maintain repopulating function of single cultured hematopoietic stem cells}

\author{
Romero Marquez, ${ }^{1,2^{\star}}$ Franziska Hettler, ${ }^{1 *}$ Renate Hausinger, ${ }^{1}$ Christina \\ Schreck, ${ }^{1}$ Theresa Landspersky, ${ }^{1}$ Lynette Henkel, ${ }^{3}$ Corinne Angerpointner, ${ }^{3}$ \\ Ihsan E. Demir, ${ }^{2}$ Matthias Schiemann, ${ }^{3}$ Florian Bassermann, ${ }^{1,4}$ Katharina S. \\ Götze,,$^{1,4}$ Rouzanna Istvánffy ${ }^{1,2 *}$ and Robert A.J. Oostendorp ${ }^{1 *}$
}

${ }^{1}$ Technical University of Munich, Klinikum Rechts der Isar, Clinic and Polyclinic for Internal Medicine III, Munich; ${ }^{2}$ Technical University of Munich, Klinikum rechts der Isar, Department of Surgery, Munich; ${ }^{3}$ Technical University of Munich, Flow Cytometry Unit of the Technical University Munich, Institute for Medical Microbiology, Immunology and Hygiene (CyTUM-MIH), Munich and ${ }^{4}$ German Cancer Consortium (DKTK), Heidelberg, Germany

*SRM and FH contributed equally as co-first authors.

${ }^{\sharp} R I$ and RAJO contributed equally as co-senior authors.

\section{ABSTRACT}

$\mathrm{H}$ ematopoietic stem cell self-renewal, proliferation, and differentiation are independently regulated by intrinsic as well as extrinsic mechanisms. We previously demonstrated that proliferation of murine hematopoietic stem cells is supported in serum-free medium supplemented with two growth factors, stem cell factor and interleukin 11. The survival of hematopoietic stem cells is additionally improved by supplementing this medium with two more growth factors, neural growth factor and collagen 1 (four growth factors) or serum-free medium conditioned by the hematopoietic stem cell-supportive stromal UG26-1B6 cells. ${ }^{1}$ Here, we describe a robust and versatile alternative source of conditioned medium from mouse embryonic fibroblasts. We found that this conditioned medium supports survival and phenotypic identity of hematopoietic stem cells, as well as cell cycle entry in single cell cultures of CD34 CD48 CD150+ Lineage $\mathrm{SCA}^{+} \mathrm{KIT}^{+}$cells supplemented with two growth factors. Strikingly, in comparison with cultures in serum-free medium with four growth factors, conditioned medium from mouse embryonic fibroblasts increased the numbers of proliferating clones and the number of Lineage $\mathrm{SCA}^{+} \mathrm{KIT}^{+}$cells, with both two and four growth factors. In addition, conditioned medium from mouse embryonic fibroblasts supported self-renewal in culture of cells with short- and long-term hematopoiesis-repopulating ability in vivo. These findings identify conditioned medium from mouse embryonic fibroblasts as a robust, alternative, serum-free source of factors to maintain self-renewal of in vivo-repopulating hematopoetic stem cells in culture.

\section{Introduction}

A major challenge in hematology is to decipher through which mechanisms and conditions hematopoietic stem cells (HSC) are maintained in the bone marrow. An additional promise of the efforts to meet this challenge is the identification of niche factors, which could be utilized to supplement protocols to expand murine and human HSC ex vivo. Although many investigators have described possible mediators of HSC maintenance in vivo, the precise factors or their combinations are still to be elucidated in detail. Also, whereas in most culture protocols, the expansion of hematopoietic colony-forming cells can readily be achieved, maintaining or expanding HSC with long-term repopulation (LTR) capacity in numbers relevant for clinical applications is a subject of intense investigation. ${ }^{2}$ Hence, there is a continued need to understand how HSC are maintained by the bone marrow niche and to determine which factors are involved.
Haematologica 2021

Volume 106(10):2633-2640

\section{Correspondence:}

ROBERT A.J. OOSTENDORP

robert.oostendorp@tum.de

Received: February 6, 2020.

Accepted: November 10, 2020.

Pre-published: November 19, 2020.

https://doi.org/10.3324/haematol.2020.249102

(C)2021 Ferrata Storti Foundation

Material published in Haematologica is covered by copyright. All rights are reserved to the Ferrata Storti Foundation. Use of published material is allowed under the following terms and conditions:

https://creativecommons.org/licenses/by-nc/4.0/legalcode. Copies of published material are allowed for personal or internal use. Sharing published material for non-commercial purposes is subject to the following conditions:

https://creativecommons.org/licenses/by-nc/4.0/legalcode, sect. 3. Reproducing and sharing published material for commercial purposes is not allowed without permission in writing from the publisher. 
In the bone marrow, the main function of the microenvironment, or niche, is to limit recruitment of HSC into the cell cycle during stress responses. However, to expand, HSC must divide and retain their LTR ability. In vivo, this process is tightly regulated, so that not all LTR-HSC are recruited and then depleted, but a significant portion of these cells return to quiescence and, thus, self-renew. Since the role of the bone marrow niche is to limit the cell cycle of HSC activation, ${ }^{3}$ it is likely that extrinsic factors govern the retention of stem cell properties of HSC during their cell cycle progression. However, the exact factors mediating these processes have, to date, not been identified.

To identify possible factors involved in the maintenance and expansion of HSC, we generated murine stromal cell lines from different tissues of the mid-gestation mouse embryo., ${ }^{4,5}$ Two of these, UG26-1B6 and EL08-1D2, support the maintenance of both murine HSC and human cord blood CD34+ ${ }^{+}$ells. ${ }^{4,5}$ Interestingly, we found that UG26-1B6 stromal cells support the maintenance of HSC in a non-contact setting, ${ }^{6,7}$ and we showed that repopulating HSC could be maintained in conditioned medium (CM) from this cell line. ${ }^{1}$ In the latter study, we further identified nerve growth factor (NGF) and collagen 1 (Col1) to be the most effective substitute for UG26-1B6-CM. The main effect of this CM seemed to be to improve survival of phenotypic HSC to almost $97 \%$ of the input cells during culture, allowing for almost complete recruitment of cells into cell division. ${ }^{1}$ In addition, the CM supported symmetrical cell division of HSC with LTR ability, which is a requirement for expansion of these cells.

In comparisons aimed at defining stem cell-maintaining factors from different stromal cells, factors characterizing the developmental origin of stromal cells should be distinguished from factors required for their HSC-maintaining function. ${ }^{8}$ A problem with this approach is that for optimal filtering for functional secreted HSC-supportive factors, multiple sources of stromal cells secreting such factors to support HSC self-renewal without the requirement of direct stroma-contact should be available. To date, only two such cell lines have been described. ${ }^{1,6,7}$ Here, we describe mouse embryonic fibroblasts (MEF) as an additional source of mid-gestation stromal cells to maintain phenotypic HSC with repopulating activity. CM from MEF (MEF-CM) has previously been used to maintain both murine and human embryonic stem cells. ${ }^{9}$ Interestingly, MEF-CM also facilitates the differentiation of embryonic stem cells into the hematopoietic lineage. ${ }^{10}$ Whether and how MEF-CM affects adult HSC and their repopulating activity under serum-free conditions remain to be established.

We here show that MEF-CM maintains the self-renewal of murine HSC with repopulating ability in single cell cultures. MEF represent a robust and easily accessible stromal cell source to study the mechanisms of the maintenance of HSC by niche factors and identify further factors supporting expansion of HSC with repopulating ability.

\section{Methods}

\section{Mice}

Eight- to 10-week old C57BL/6.J (B6; CD45.2), B6.SJLPtprca.Pep3b/BoyJ (Ly5.1; CD45.1), 129S2/SvPasCrl (129; CD45.2) mice were obtained from Charles River Laboratories (Lyon, France). All experiments were approved by the Government of Upper Bavaria. Animals were housed in micro-isolators under spe- cific pathogen-free conditions, according to Federation of Laboratory Animal Science Associations and institutional recommendations.

\section{Primary cells and mouse embryonic fibroblasts}

MEF were generated from embryos (E11.5 or E13.5) using a generic protocol ${ }^{11}$ without trypsin digestion of the embryonic tissue. The dissected embryos were cultured in MEF medium (see Online Supplementary Methods) at $37^{\circ} \mathrm{C}$ and passaged four times every 3 to 4 days.

\section{Generation of conditioned medium}

$\mathrm{CM}$ was prepared essentially as described previously. ${ }^{1}$ In brief, $70,000 / \mathrm{cm}^{2} \mathrm{MEF}$ (passage 4) were cultured to near confluence in MEF medium (for details see Online Supplementary Methods). Twelve hours later the cells were $\gamma$-irradiated (30 Gy X-Ray; Gulmay Type RS225), washed twice with Dulbecco phosphatebuffered saline (DPBS; Gibco) and cultured for $72 \mathrm{~h}$ with serumfree medium (SFM; StemSpan, Stemcell Technologies).

\section{Flow cytometry analyses}

Surface antigens were stained with antibodies from either Invitrogen- or eBioscience-ThermoFisher (Online Supplementary Table S1). Flow cytometry was performed on a CyAn ADP Lx P8; cell sorting was performed using an Astrios high speed sorter (both from Beckman-Coulter). Data were analyzed with FlowJo software (TreeStar).

\section{Cell sorting and single cell cultures}

For single cell cultures, single murine CD34- CD48 CD150+ Lineage SCA1+ $\mathrm{KIT}^{+}$(LSK) cells (CD34- SLAM cells) were sorted directly into a 96-well round-bottomed plate on an Astrios high speed cell sorter (Beckman Coulter). Single cells were deposited with an efficiency of $90 \%$. These CD34 SLAM cells comprise both CD34 and CD34 $4^{\text {low }}$ populations, as these have been reported to contain similar frequencies of long-term repopulating cells. ${ }^{12}$ These plates were preloaded with $100 \mu \mathrm{L}$ of filtered $(0.20 \mu \mathrm{m}$, Sartorius) CM supplemented with murine stem cell factor (SCF: $100 \mathrm{ng} / \mathrm{mL}$ ) and murine interleukin-11 (IL-11; $20 \mathrm{ng} / \mathrm{mL}$ ) (two growth factors; 2GF), both from R\&D Systems (BioTechne). Additional cultures were further supplemented with Col1 (300 $\mu \mathrm{g} / \mathrm{mL}$; BioVendor) and human NGF (250 ng/mL; R\&D SystemsBioTechne) (four growth factors; 4GF). Every 24 h, the number of cells per well was enumerated using a light microscope. After 5 days, cells that had divided at least once were harvested, pooled, stained with antibodies, and analyzed by flow cytometry. In some experiments, colony-forming ability of pools from divided cells was assessed using standard assays (M3434; Stemcell Technologies).

\section{In vivo transplantation assay}

An in vivo repopulation assay using competitive transplantation was performed as described previously. ${ }^{13,14}$ For this purpose, 20 wells showing at least one cell division of CD34- SLAM cells from 129xLy5.1 F1 (129Ly5.1; CD45.1xCD45.2) donors were harvested, pooled, and transplanted into lethally irradiated 129xB6 F1 (129B6; CD45.2) recipient mice, together with $1 \times 10^{5}$ bone marrow cells and $5 \times 10^{5}$ spleen cells from $129 \mathrm{~B} 6$ mice. Donor cell engraftment was analyzed every 5, 10 and 16 weeks in the peripheral blood and 16 weeks after transplantation in the bone marrow cells, as described previously. ${ }^{13,14}$

\section{Apoptosis assay}

Apoptosis was determined in $48 \mathrm{~h}$ cultures of LSK cells in 12well plates, prefilled with $2 \mathrm{~mL}$ SFM or MEF-CM with the growth 
factors indicated. After culture, LSK cells were permeabilized and stained using the FITC Annexin V Apoptosis Detection Kit I according to the manufacturer's instructions (BD-Biosciences).

\section{Statistical evaluation}

For statistical evaluation of the experiments, the non-parametric Mann-Whitney U test was used (Prism, GraphPad Software). A P value $<0.05$ was considered statistically significant. Data are presented as dot plots of columns with means \pm standard deviation.

\section{Results}

Here, we describe cultures in which sorted single CD34 SLAM cells from the bone marrow of B6 or 129Ly5.1 mice were cultured either in SFM, or in serum-free MEF-CM supplemented with either 4GF (SCF, IL-11, NGF, and Col1) or 2GF (SCF and IL-11). The single cell cultures were monitored for the presence and number of cells each day for a total of 5 days (Figure 1A). These experiments showed that in SFM 4GF the number of wells with dividing cells was increased compared to those in SFM 2GF (60\% vs. $42 \%$, respectively) (Figure 1B). Although in the current experiments SFM 4GF yielded lower survival than previously reported, ${ }^{1}$ our results show that the extra addition of NGF and Col1 to SFM 2GF improved survival of murine CD34SLAM cells.

To further define stromal cell factors substituting $\mathrm{CM}$ in HSC cultures, we realized that additional sources of CM will be needed to optimally define factors maintaining HSC self-renewal. Hence, we chose to study a robust, readily available and non-transformed source of mid-gestation embryonic stromal cells: MEF. Considering that UG26-1B6 was the only cell line consistently supporting HSC in noncontact cultures, ${ }^{6,7}$ we not only added 2 GF but also explored adding 4GF to MEF-CM. In a first series of experiments, we found that CM from E11.5 or E13.5 MEF with 4GF supported cell cycle recruitment of single CD34 SLAM cells to a similar extent (Online Supplementary Figure S1A). Since E13.5 embryos used for support of embryonic stem cells are typically isolated at E13-E14, ${ }^{15}$ consistently yield more cells than E11.5 embryos, and MEF-CM from both sources behaved similarly, we performed all following experiments with E13.5 MEF-CM. To assess the variability of different MEF-CM, we prepared MEF from seven individual embryos and found that although at the beginning of culture recruitment into cell division varied among different individual MEF-CM preparations, 4 and 5 days after the start of cultures, the growth kinetics were remarkably consistent (Online Supplementary Figure S1B).

To further optimize cell cultures, we compared CM generated from freshly isolated MEF or from previously frozen MEF. Here we found that CM from both fresh and previously frozen MEF stimulated cell cycle recruitment and maintenance of proliferation to a similar extent (Online Supplementary Figure S2A). Another important issue was to consider whether CD34 SLAM cells from different mouse strains we routinely use in transplantation assays ${ }^{3,1,1,16}$ would differ in culture with MEF-CM. We found that CD34SLAM cells isolated either from B6 or 129Ly5.1 mice showed indistinguishable results in terms of proliferation and the number of dividing cells (Online Supplementary Figure S2B).

When comparing single cell cultures with MEF-CM 2GF and 4GF, we found that although survival was similar in
MEF-CM with 2GF or 4GF (Figure 1C, D), MEF-CM 2GF stimulated the cell cycle more efficiently compared to MEFCM 4GF (Figure 1E). Indeed, cultures with MEF-CM 2GF showed larger clones at days 2 and 3 of culture (Figures 1C, E), while the time to first division was similarly shortened (Figure 1F), resulting in comparable increased clone sizes (Figure 1G) for both MEF-CM conditions compared to SFM 4GF. Overall, the percentage of wells with cells, wells with dividing cells, and mean clone size were significantly increased when using MEF-CM with either $2 \mathrm{GF}$ or $4 \mathrm{GF}$ as compared with SFM 4GF (Figure 1C-E, G).

To study whether these differences in number of dividing cells were attributable to cell death, we determined apoptosis in cultures of LSK cells under SFM 4GF, MEF-CM 2GF or MEF-CM 4GF conditions. These experiments show that after $48 \mathrm{~h}$ of bulk culture, LSK cells cultured in MEF-CM 2GF maintained a higher percentage of viable cells and a lower percentage of apoptotic cells compared with cells cultured in MEF-CM 4GF or SFM 4GF, confirming the positive effect of MEF-CM for survival (Figure 2A, B).

Flow cytometric analyses of pooled clones from single cell cultures may reveal patterns of differentiation behavior as well as retention of LSK phenotypes. ${ }^{16}$ Our experiments showed that the relative numbers of $\mathrm{CD} 11 \mathrm{~b}^{+} \mathrm{Gr} 1^{\text {med }}$ and $\mathrm{Gr}^{\mathrm{hi}}$ myeloid cells were unchanged in all three culture conditions (Figure 2C, D, Online Supplementary Figure S3 for isotype controls). However, the number of LSK cells recovered after 5 days of single cell culture was consistently higher in cultures with MEF-CM 2GF (Figure 2E, 2F). In addition, cells from MEF-CM 2GF cultures showed a significantly increased number of colonies in semi-solid medium compared with those from MEF-CM 4GF and SFM 4GF cultures (Figure 2G). These results suggest that whereas myeloid differentiation progresses at similar rates under all culture conditions, MEF-CM 2GF appears to maintain the HSC phenotype and progenitor function best in culture.

To determine whether this culture condition would also support self-renewal of murine CD34 SLAM cells with repopulating ability, we collected wells containing divided cells after 5 days of culture and transplanted pools of 20 of these clones per lethally irradiated recipient (Figure 3A). Analysis of donor engraftment in the peripheral blood after 5,10 and 16 weeks showed increased engraftment of donor cells from cultures in MEF-CM 2GF as compared to the MEF-CM 4GF or SFM 4GF-cultured CD34 SLAM cells (Figure 3B). At the early 5-week timepoint, engraftment from MEF-CM 2GF cultures was particularly prominent, suggestive of production of short-term repopulating cells in culture, followed by a stable level of engraftment at later time points (Figure 3B). With regard to differentiation of the transplanted clones, donor HSC cultured in either MEF-CM 4GF or 2GF showed higher lymphoid engraftment throughout the 16-week observation period (Figure 3C). Furthermore, donor HSC cultured in MEF-CM 2GF showed a high level of myeloid engraftment at all time points as compared with HSC cultured in MEF-CM 4GF or SFM 4GF (Figure 3D).

As in peripheral blood, donor engraftment in the bone marrow was highest in mice repopulated with divided donor CD34 SLAM cells from cultures in MEF-CM 2GF (Figure 3E, Online Supplementary Figure S4A). More importantly, these mice also showed the highest regeneration of the donor-derived CD48 LSK cells (Figure 3F). Compared to mice receiving cells from SFM 4GF cultures, both cultures under MEF-CM 4GF and 2GF conditions showed higher 
A

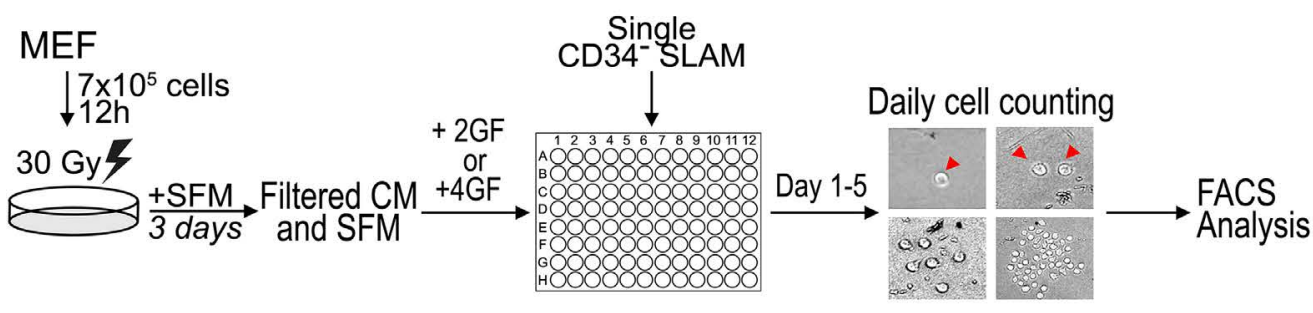

B

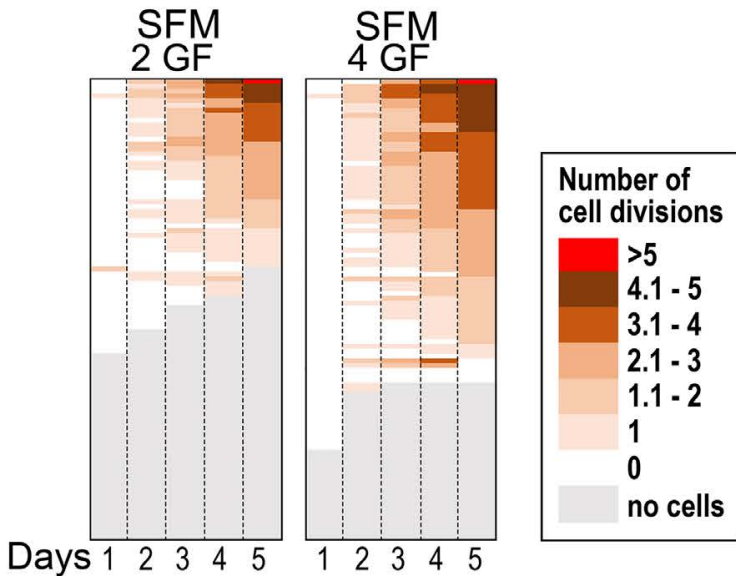

C
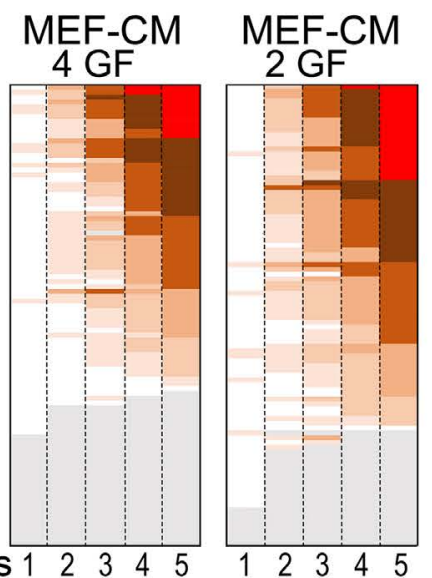

D

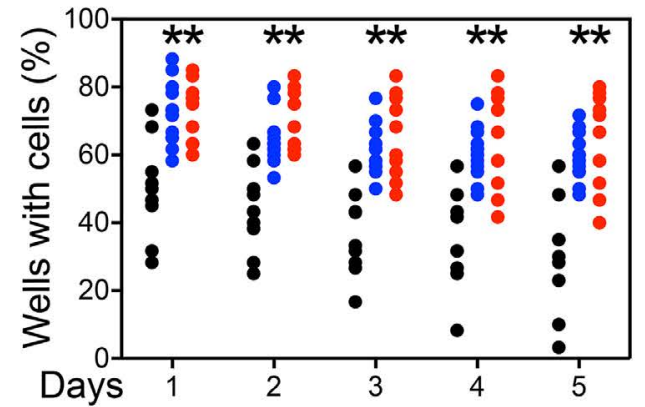

$\mathrm{E}$

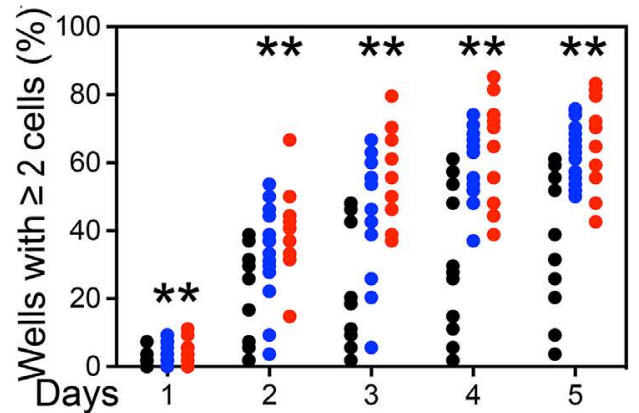

F

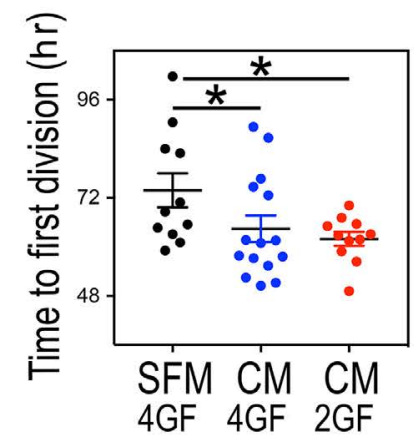

G

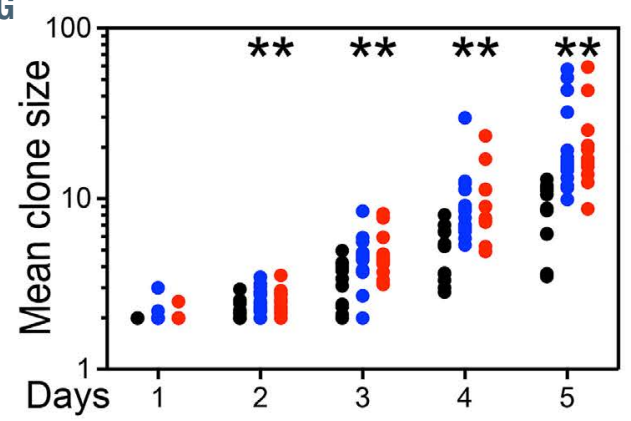

-SFM 4GF - CM 4GF - CM 2GF

Figure 1. Single cell cultures in serum-free medium and mouse embryonic fibroblast-conditioned medium. (A) Experimental design: single CD34 SLAM cells from 129Ly5.1 mice were sorted into a 96-well round-bottomed plate with SFM and MEF-CM, supplemented with either murine SCF (100 ng/mL) and murine IL-11 (20 ng/mL) alone (2GF) or with additional human NGF (250 ng/mL) and Col1 (100 ng/mL) (4GF). Cells were microscopically inspected and counted every $24 \mathrm{~h}$ for $5 \mathrm{days}$. (B) Heat maps of the number of calculated cell divisions per well for each CD34 SLAM cell at each day of culture. Left heat map: culture in SFM 2GF; right heat map: culture in SFM 4GF. Each row represents cell divisions of a single cell in a single well. (C) Heat maps of the number of calculated cell divisions per well for each CD34 SLAM cell at each day of culture. Left heat map: culture in MEF-CM 4GF; right heat map: culture in MEF-CM 2GF. Again, each row represents cell divisions of a single cell in a single well. (D) Percentage of wells with detectable cells per day from each plate cultured in SFM 4GF, MEF-CM 4GF, and MEF-CM 2GF. (E) Percentage of wells with divided cells ( $\geq 2$ cells) from each plate cultured in SFM 4GF, MEF-CM 4GF, and MEF-CM 2GF. (F) Mean hours of time to first division of cells cultured in SFM 4GF, MEF-CM 4GF, and MEF-CM 2GF. (G) Mean clone size per day from each plate cultured in SFM 4GF, MEF-CM 4GF, and MEF-CM 2GF. Representative examples of two plates are shown in (B) and (C). Panels (D-G) show the results of at least four independent experiments with separate donor mice totalling: $n=11$ for SFM $4 G F$, $n=15$ for MEF-CM $4 G F$, and $\mathrm{n}=11$ for MEF-CM 2GF. In all graphs, black dots represent results for SFM 4GF, blue dots represent results for MEF-CM 4GF and red dots represent results for MEF-CM 2GF. * $P<0.05$ using the non-parametric Mann-Whitney U-test of comparisons between SFM 4GF and either MEF-CM 4GF or MEF-CM 2GF. MEF: mouse embryonic fibroblasts; SFM: serum-free medium; CM: conditioned medium; 2GF: two growth factors (i.e., stem cell factor [SCF] and interleukin-11 [IL-11]); 4GF: four growth factors (i.e., SCF, IL-11, nerve growth factor [NGF] and collagen 1 [Col1]); SLAM: CD48 CD150+ Lineage SCA1 ${ }^{+} \mathrm{KIT}^{+}$; FACS: fluorescence activated cell sorting. 
generation of the donor pool of short- and LTR cellenriched fractions of $\mathrm{CD}_{4} 4^{+}$and CD34 CD48 LSK cells (Figure 3G, Online Supplementary Figure $S 4 B, C$ ), respectively. However, the total number of CD34 CD48 LSK cells was higher in mice receiving cells from MEF-CM 2GF cultures than in those receiving cells from MEF-CM 4GF and SFM 4GF cultures (Figure 3G).

\section{Discussion}

Some of the biggest challenges in hematology are to understand how niche cells regulate self-renewal and survival of HSC and to develop methods with which HSC can be robustly and reproducibly expanded in vitro. In the current work, we used MEF as a source of secreted HSC-regulatory molecules and found that CM prepared from these stromal cells promoted the survival and self-renewal of murine HSC in culture. MEF are derived from primary material, which can be easily generated, even from most mouse mutants causing embryonic lethality, and used by many investigators to study mechanisms of embryonic stem cell regulation. Here, we demonstrate that MEF are a robust alternative in protocols aimed at maintaining and expanding HSC. Furthermore, MEF can be used to identify secreted factors involved in HSC maintenance. We show that MEF-CM not only supports the survival of HSCenriched CD34 SLAM cells in cultures supplemented with 2GF, but also improves their self-renewal under both $2 \mathrm{GF}$ and $4 \mathrm{GF}$ conditions.

The additional use of MEF-CM prepared under serumfree conditions improves survival and self-renewal of CD34 SLAM cells. In this respect, MEF-CM performs the same survival-promoting function as the CM from the stromal cell line UG26-1B6 that we studied previously. ${ }^{1}$ As in our previous study, the stromal CM did not seem to affect
A

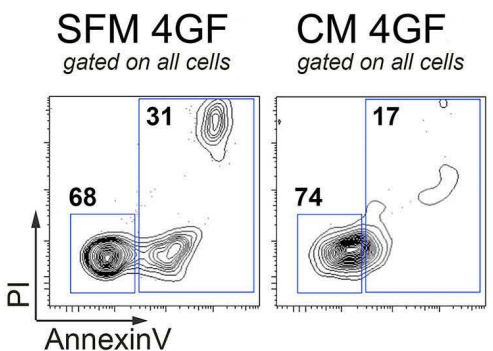

C

SFM 4GF $\quad$ CM 4GF $\quad$ CM 2GF

gated on non-lymphoid gated on non-lymphoid gated on non-lymphoid
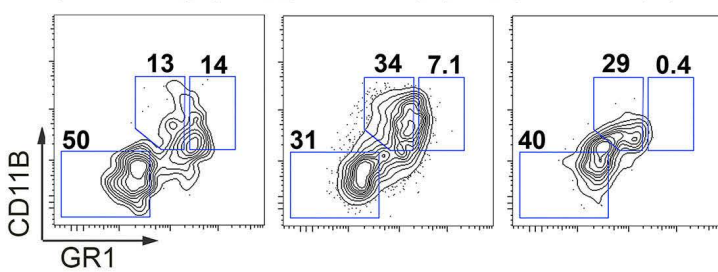

gated on all cells

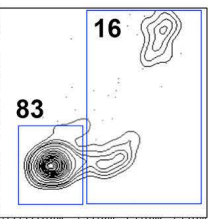

E

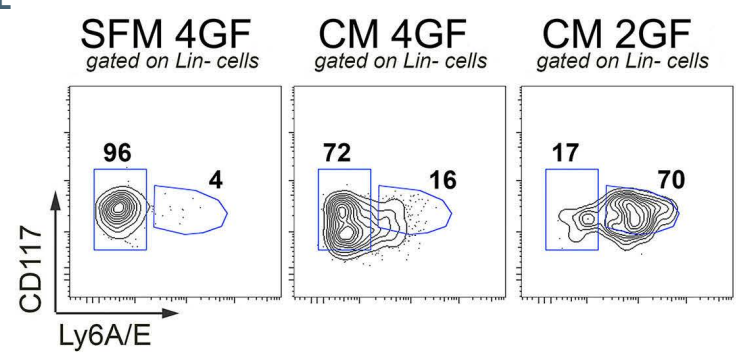

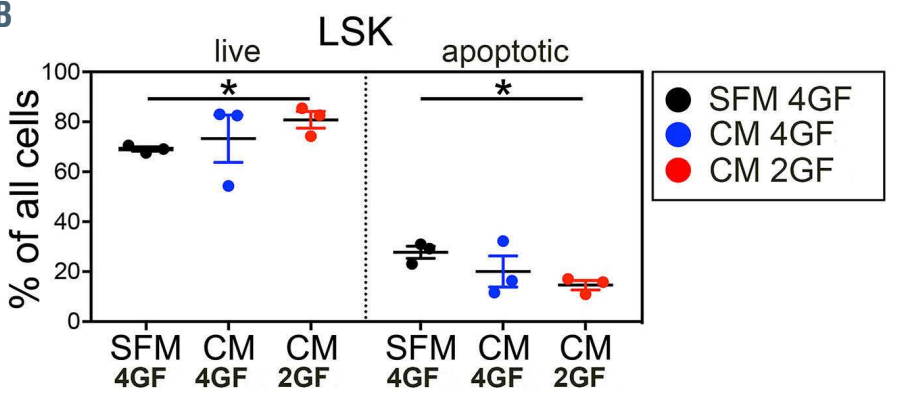

D
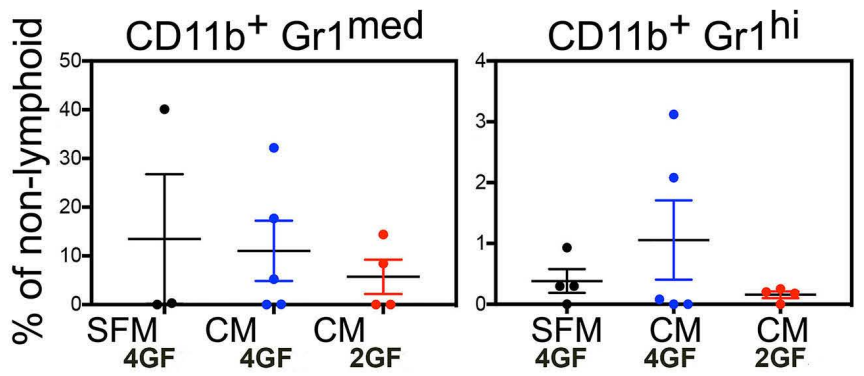

$F$

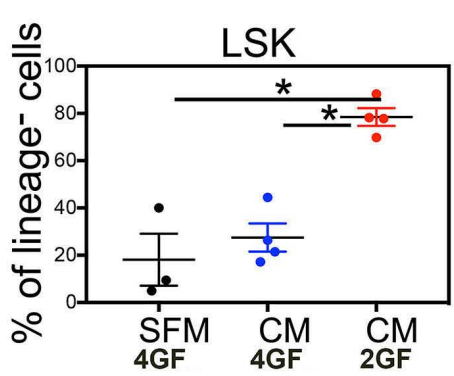

G

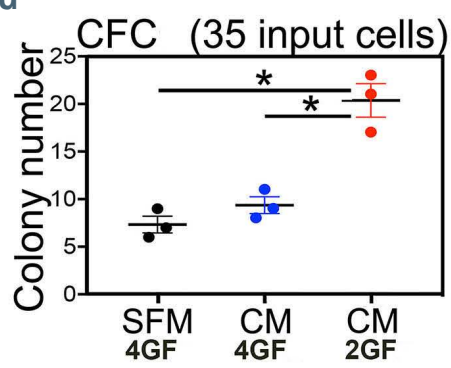

Figure 2. Maintenance of LSK cells and differentiation of CD34 SLAM cells in single cell cultures. Cells were cultured in either SFM 4GF, MEF-CM 4GF, or MEF-CM 2GF as indicated. (A) Representative flow cytometric plots of annexin V and propidium iodide (PI) staining of sorted LSK cells cultured for $48 \mathrm{~h}$. (B) Percentage of viable (annexin $\mathrm{V} \cdot \mathrm{Pl}$ ) and apoptotic (annexin $\mathrm{V}^{+}$) LSK cells after $48 \mathrm{~h}$ of culture. (C) Representative flow cytometric plots of non-lymphoid cells stained for myeloid markers CD11b and Gr1. (D) Percentage of CD11 $b^{+}$Gr1 $1^{\text {med }}$ monocytic cells (left plot) and CD11 $b^{+}$Gr1 $1^{\text {hi }}$ granulocytic cells (right plot) after 5 days of culture. (E) Representative flow cytometry plots of cultured CD34 SLAM cells stained for lineage markers (CD3E, CD11b CD45R Gr1), and additionally stained for KIT and SCA1. (F) Percentage of LSK cells 5 days after culture of CD34 SLAM cells. (G) Total numbers of CFC, 5 days after culture of CD34SLAM cells. The experiments in panels (B) and $(G)$ were performed independently three times with one 96-well plate for each condition per experiment. The experiments in panels (C-F) were performed independently three times with a total of either four (SFM 4GF, MEF-CM 2GF) or five (MEF-CM 4GF) 96-well plates evaluated. As in Figure 1, black dots represent results for SFM 4GF, blue dots represent results for MEF-CM 4GF and red dots represent results for MEF-CM 2GF. $* P<0.05$ using the non-parametric Mann Whiney U-test for comparisons between SFM 4GF and either of the two MEF-CM conditions. MEF: mouse embryonic fibroblasts; SFM: serum-free medium; CM: conditioned medium; 2GF: two growth factors (i.e., stem cell factor [SCF] and interleukin-11 [IL-11]); 4GF: four growth factors (i.e., SCF, IL-11, nerve growth factor [NGF] and collagen 1 [Col1]); SLAM: CD48 CD150+ Lineage SCA1 $1^{+}$KIT'; LSK: Lineage SCA1+ KIT+; CFC: colony-forming cells. 
proliferation rate. However, compared to the serum-free cultures, the MEF-CM shortened the time to first division. As such, the proliferation process as a whole was affected in that larger clones were detected after 5 days. The processes of survival, cell cycle (recruitment), and selfrenewal are not only independently regulated in murine HSC, ${ }^{1}$ but also in human HSC. ${ }^{17}$ Thus, similarly to the UG26-1B6-derived CM, MEF-CM can be used to define stromal factors beneficial for survival and self-renewal of murine HSC, and possibly also human HSC.

In our previous study, we showed that some of those fac- tors, in particular NGF and Col1, may substitute for the UG26-1B6-CM. ${ }^{1}$ Indeed, in the present study, culture of CD34 SLAM cells in SFM 4GF improved survival over cultures in SFM 2GF. Importantly, the MEF-CM 2GF condition promoted both survival and cell cycle recruitment of single CD34 SLAM cells compared to SFM 4GF. It is also noteworthy that cultures in MEF-CM 2GF produced significantly more repopulating cells and maintained cells repopulating hematopoiesis at significantly higher levels throughout the engraftment period. The addition of extra NGF and Col1 to MEF-CM 2GF cultures did not additionally improve

A
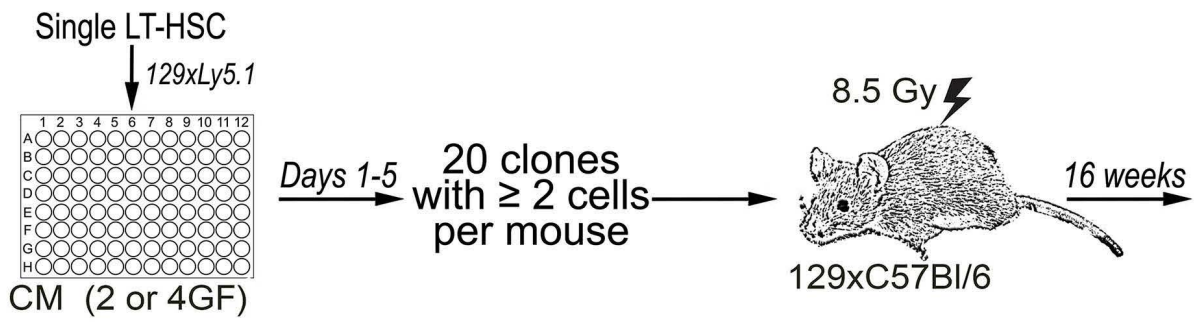

FACS analysis

SFM 4 GF - CM 4GF CM 2GF

\section{Donor PB engraftment}

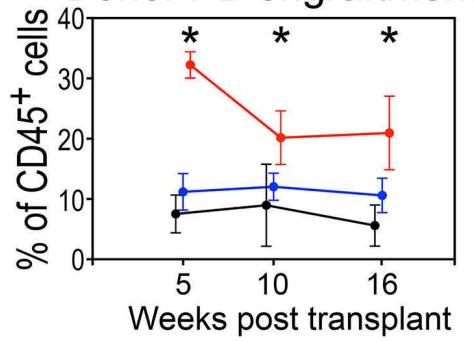

E

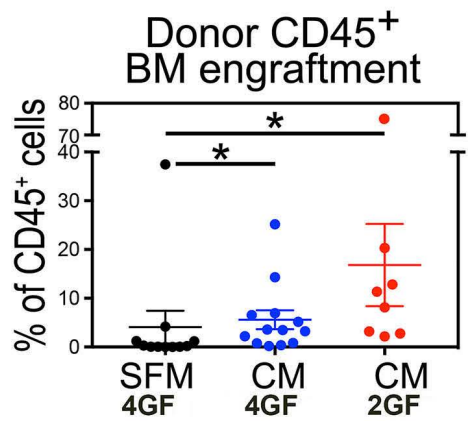

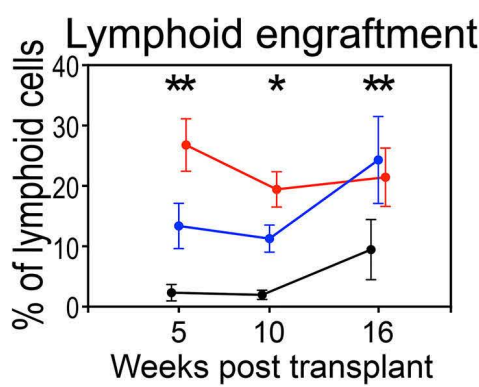

$\mathrm{F}$

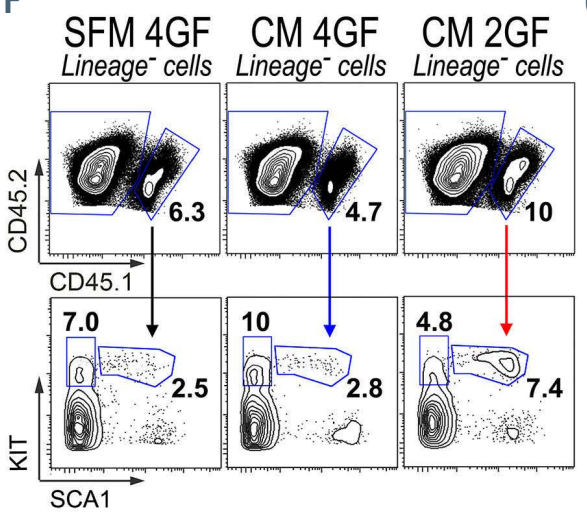

D

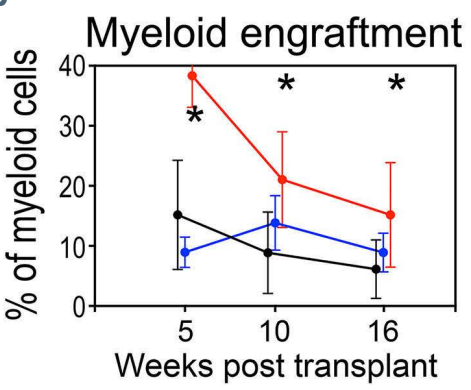

G

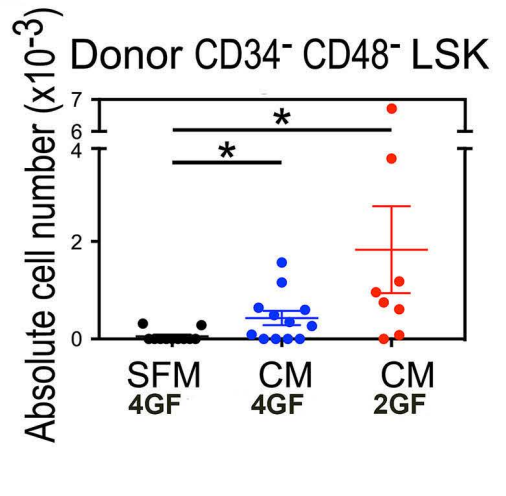

Figure 3. Self-renewal of repopulating hematopoietic stem cells in single cell cultures. (A) Experimental design: CD34 SLAM cells (positive for both CD45.1 and CD45.2) were sorted as single cells into 96-round-bottomed plates prefilled with SFM 4GF, MEF-CM 4GF, or MEF-CM 2GF as specified in Figure 1. To determine selfrenewal of repopulating cells in cultures of single cells, 20 wells in which at least one cell division had occurred (cell clones) were harvested and transplanted into lethally irradiated (CD45.2) primary recipients together with competitor cells (CD45.2) as described in the Methods section. (B) Donor cell engraftment in peripheral blood, 5, 10, and 16 weeks after transplantation of divided CD34 SLAM cells previously cultured under the specified conditions. (C) Percentages of the lymphoid $\left(\mathrm{CD} 3 \mathrm{e}+\right.$ and $\mathrm{CD} 45 \mathrm{R}(\mathrm{B} 220)+$ cells) and (D) myeloid $\left(\mathrm{CD} 11 \mathrm{~b}^{+} \mathrm{Gr} 1^{+}\right)$engraftment in peripheral blood, 5, 10, and 16 weeks after transplantation of growing clones from 5 -day cultures as fractions of the total (donor + recipient $=100 \%$ ) CD45 lymphoid and myeloid compartments, respectively. (E) Percent donor engraftment in CD45 cells of the bone marrow after transplantation of divided cells from single cell cultures. (F) Representative flow cytometric plots of donor and recipient cells in the Lineage cell fraction from the bone marrow (upper row) and the donor-derived Lineage fraction (lower row), showing myeloid progenitors (SCA1 KIT') cells and LSK cells (Lineage SCA1 ${ }^{+} \mathrm{KIT}^{+}$cells; lower row), 16 weeks after transplantation. (G) Absolute number of HSC-enriched CD34 CD48 LSK donor cells from the bone marrow of mice transplanted with 20 clones each from single cell cultures. For gating of CD34 CD48 LSK cells from the LSK cells shown in the previous panel (F), see Online Supplementary Figure S4C. The absolute number of cells was calculated by multiplying relative numbers of Lineage and LSK cells by the total number of donor cells in the bone marrow (Online Supplementary Figure S4A) The results shown here represent three independent experiments with a total of 11 recipients transplanted with divided cells from cultures in SFM 4GF, 13 recipients transplanted with divided cells from cultures in MEF-CM 4GF, and 8 recipients with divided cells from cultures in MEF-CM 2GF. As in Figure 1, black dots represent results for SFM 4GF, blue dots represent results for MEF-CM 4GF and red dots represent results for MEFCM 2GF. $* P<0.05$ using the non-parametric Mann Whitney U-test for comparisons between SFM 4GF and either of the two MEF-CM conditions. LT-HSC: long-term hematopoietic stem cells; CM: conditioned medium; 2GF: two growth factors (i.e., stem cell factor [SCF] and interleukin-11 [IL-11]); 4GF: four growth factors (i.e., SCF, IL-11, nerve growth factor [NGF] and collagen 1 [Col1]); SFM: serum-free medium; FACS: fluorescence activated cell sorting; PB: peripheral blood; BM: bone marrow; LSK: Lineage SCA1 ${ }^{+} \mathrm{KIT}^{+}$. 
survival, or the maintenance of LSK cells in culture or repopulating activity of growing clones. This suggests that although NGF and Col1 improve HSC survival in culture, the two factors do not cooperate or synergize with other MEF-CM factors in maintaining or expanding HSC with repopulating ability.

MEF-CM has previously been used to maintain murine embryonic stem cells. ${ }^{9}$ A proteomic study of serum-free MEF-CM showed a considerable amount of different collagens - types $\alpha 1$ (I, II, and IV), $\alpha 2$ (I), pro-collagens (type Va2), and collagen remodeling enzymes (MMP-2 and -3, PCOLCE, and TIMP2), ${ }^{18}$ suggesting that perhaps adding more collagen could be counter-productive. The effects of different concentrations of soluble collagen on the production of short- and long-term repopulating cells have, as far as we know, not been explored. However, NGF was not detected in this study of MEF-CM, ${ }^{18}$ suggesting a low concentration of this cytokine or that other factors in the MEFCM can substitute for NGF. Thus, it is likely that in combination with MEF-CM, the precise concentrations of NGFlike activity and Col1 are critical in collaborating with other soluble factors to promote survival and self-renewal. These results indicate that precise titration of growth factors and collagen concentrations and assessment of collaborative and synergistic interactions with other stromal factors in the MEF-CM are needed, as noted in other studies. ${ }^{19,20}$

In order to use MEF-CM in protocols for maintaining or expanding HSC, the MEF and their CM need to be defined precisely, particularly to minimize batch-to-batch variation. Indeed, although MEF have been considered homogeneous, it has been noted that within the morphologically indistinguishable fibroblasts, cells with different potentials may be present. ${ }^{21}$ Yet, our study shows that MEF-CM prepared from fresh MEF or previously frozen samples of MEF showed minimal variation in their support of proliferation and survival of HSC. The precise form in which the supportive factors are presented to the HSC remains to be established. We harvest serum-free medium from MEF monolayers incubated with SFM. Thus, our MEF-CM will not only include secreted factors, but also extracellular matrix, matricellular proteins, and extracellular vesicles, which may carry mRNA and regulatory micro-RNA. Indeed, the matricellular protein CCN2 has been shown to be important for HSC self-renewal, ${ }^{14}$ and purified extracellular vesicles from different sources have been used to support growth of both murine ${ }^{22}$ and human HSC. ${ }^{23,24}$ Thus, it will be of interest in future studies to dissect the identity of HSC-supportive factors in the different structures included in the CM.

Our study defines a novel source of stromal cells capable of maintaining repopulating HSC in vitro under non-contact conditions. In previous studies, we used different cell lines to filter cell-type-specific factors and determine stromal factors required for HSC in co-cultures. ${ }^{6}$ These efforts yielded several new mediators of HSC self-renewal under stress conditions in vivo., ${ }^{3,16,25}$ Although these studies are helping to dissect molecular mechanisms in the stromal cell-mediated regulation of HSC, only the UG26-1B6 and EL08-1D2 (with extra addition of WNT5A) cell lines were consistently shown to maintain HSC under non-contact conditions. ${ }^{1,6,7}$ Thus, together with these stromal lines, MEF can be used to better define soluble factors critical for maintaining repopulating activity in vitro, perhaps by combined analyses as we have previously described for HSC-supportive stromal cells requiring direct contact with HSC. ${ }^{8}$ Such knowledge will be critical to improve protocols aimed at maintaining or expanding HSC in vitro.

Current knowledge of such expansion protocols have shown that precise titration of SCF, IL-1120 and thrombopoietin, ${ }^{26-28}$ are critical for successful maintenance of LTR-HSC activity. Moreover, since variations in batches of bovine serum albumin (BSA) in SFM have been shown to affect culture outcomes, ${ }^{29}$ replacement of BSA with hemopexin ${ }^{29}$ or polyvinyl alcohol may lead to more consistent results in HSC cultures. ${ }^{26}$ In addition, new approaches are aiming to replace cytokines by pharmacological substances directly modulating critical signaling pathways to inhibit HSC differentiation, such as MEK inhibitors, ${ }^{30}$ arylhydrocarbon receptor antagonists, ${ }^{31,32}$ or epigenetic regulators. ${ }^{33}$ Using these cytokines and substances, expansions of murine LTR-HSC numbers by an estimated 10-fold in 10 days $^{29}$ and 236- to 899-fold in 28 days ${ }^{26}$ have been described. Although we did not quantify the number of HSC in our experiments, we could assume a moderate expansion of 3 -fold after 5 days from our transplantation experiments. If we further assume that the expanding pool of differentiating cells would not affect the number of LTR-HSC generated, ${ }^{34}$ culture in MEF-CM 2GF could result in 9-fold expansion after 10 days and over 200-fold expansion after 25 days. Thus, even moderate improvements in expansion suggest that combining factors isolated from MEF-CM with BSA replacement agents or pharmacological inhibitors may be viable strategies to improve HSC expansion protocols.

In summary, our results show that cultures of HSC in E13.5 MEF-CM 2GF support induction and propagation of cell division, as well as survival of individual HSC. More importantly, MEF-CM supports self-renewal of LTR-HSC in culture. Since MEF are easily generated, renewable and can be genetically modified, MEF and their CM represent promising tools in defining novel secreted stromal factors for promoting survival, cell division and self-renewal of HSC, which may help to improve in vitro expansion protocols and HSC engraftment in vivo. Furthermore, such studies may facilitate the understanding of the mechanistic basis of how stromal cells maintain HSC in the niche.

\section{Disclosures}

No conflicts of interest to disclose.

\section{Contributions}

FH, SRM, RH, TS and CS performed experiments; FH, SRM and RI collected and analyzed data; $L H, C A$ and MS sorted cells; $K S G, I E D$ and FB provided infrastructure and contributed critical reagents, materials or analytic tools; $R I$ and RAJO designed research; FH, SRM, RI and RAJO wrote the manuscript.

\section{Acknowledgments}

We thank the animal caretakers of the Center for Preclinical Research (ZPF, TranslaTUM, Klinikum rechts der Isar) for excellent animal care.

\section{Funding}

This work was supported by the German Research Council (Deutsche Forschungsgemeinschaft: DFG) (SFB 1243 [project A09]; $O O$ 8/16, and FOR 2033 [project B3]) and the German "José Carreras Leukemia" Foundation (DJCLS) through funds to KSG (R14/18). 


\section{References}

1. Wohrer S, Knapp DJ, Copley MR, et al. Distinct stromal cell factor combinations can separately control hematopoietic stem cell survival, proliferation, and self-renewal. Cell Rep. 2014;7(6):1956-1967.

2. Papa L, Djedaini M, Hoffman R. Ex vivo HSC expansion challenges the paradigm of unidirectional human hematopoiesis. Ann N Y Acad Sci. 2020;1466(1):39-50.

3. Ruf F, Schreck C, Wagner A, et al. Loss of Sfrp2 in the niche amplifies stress-induced cellular responses, and impairs the in vivo regeneration of the hematopoietic stem cell pool. Stem Cells. 2016;34(9):2381-2392.

4. Oostendorp RAJ. Stromal cell lines from mouse aorta-gonads-mesonephros subregions are potent supporters of hematopoietic stem cell activity. Blood. 2002;99(4): 1183-1189.

5. Oostendorp RAJ. Embryonal subregionderived stromal cell lines from novel temperature-sensitive SV40 T antigen transgenic mice support hematopoiesis. J Cell Sci. 2002;115(10):2099-2108.

6. Oostendorp RA, Robin C, Steinhoff C, et al. Long-term maintenance of hematopoietic stem cells does not require contact with embryo-derived stromal cells in cocultures. Stem Cells. 2005;23(6):842-851.

7. Buckley SM, Ulloa-Montoya F, Abts D, et al. Maintenance of HSC by Wnt5a secreting AGM-derived stromal cell line. Exp Hematol. 2011;39(1):114-123.

8. Desterke C, Petit L, Sella N, et al. Inferring gene networks in bone marrow hematopoietic stem cell-supporting stromal niche populations. iScience. 2020;23(6): 101222 .

9. Xu C, Inokuma MS, Denham J, et al. Feeder-free growth of undifferentiated human embryonic stem cells. Nat Biotechnol. 2001;19(10):971-974.

10. Lee JB, Graham M, Collins TJ, et al. Reversible lineage-specific priming of human embryonic stem cells can be exploited to optimize the yield of differentiated cells. Stem Cells. 2015;33(4):11421152.

11. Durkin ME, Qian X, Popescu NC, Lowy DR. Isolation of mouse embryo fibroblasts. Bio Protoc. 2013;3(18):e908.

12. Osawa M, Hanada K, Hamada $H$, Nakauchi H. Long-term lymphohematopoietic reconstitution by a single
CD34-low/negative hematopoietic stem cell. Science. 1996;273(5272):242-245.

13. Istvanffy R, Kroger M, Eckl C, et al. Stromal pleiotrophin regulates repopulation behavior of hematopoietic stem cells. Blood. 2011;118(10):2712-2722.

14. Istvanffy R, Vilne B, Schreck C, et al. Stroma-derived connective tissue growth factor maintains cell cycle progression and repopulation activity of hematopoietic stem cells in vitro. Stem Cell Reports. 2015;5(5):702-715.

15. McElroy SL, Reijo Pera RA. Culturing human embryonic stem cells with mouse embryonic fibroblast feeder cells. CSH Protoc. 2008;2008:pdb.prot5042.

16. Schreck C, Istvanffy R, Ziegenhain C, et al. Niche WNT5A regulates the actin cytoskeleton during regeneration of hematopoietic stem cells. J Exp Med. 2017; 214(1):165-181.

17. Knapp DJ, Hammond CA, Miller PH, et al. Dissociation of survival, proliferation, and state control in human hematopoietic stem cells. Stem Cell Rep. 2017;8(1):152-162.

18. Lim JW, Bodnar A. Proteome analysis of conditioned medium from mouse embryonic fibroblast feeder layers which support the growth of human embryonic stem cells. Proteomics. 2002;2(9):1187-1203.

19. Zandstra PW, Conneally E, Petzer AL, Piret JM, Eaves CJ. Cytokine manipulation of primitive human hematopoietic cell selfrenewal. Proc Natl Acad Sci U S A. 1997;94(9):4698-4703.

20. Audet J, Miller CL, Eaves CJ, Piret JM. Common and distinct features of cytokine effects on hematopoietic stem and progenitor cells revealed by dose-response surface analysis. Biotechnol Bioeng. 2002;80(4): 393-404

21. Singhal PK, Sassi S, Lan L, et al. Mouse embryonic fibroblasts exhibit extensive developmental and phenotypic diversity. Proc Natl Acad Sci U S A. 2016;113(1):122127.

22. Stik G, Crequit S, Petit L, et al. Extracellular vesicles of stromal origin target and support hematopoietic stem and progenitor cells. J Cell Biol. 2017;216(7):2217-2230.

23. Morhayim J, Ghebes CA, Erkeland SJ, et al. Identification of osteolineage cell-derived extracellular vesicle cargo implicated in hematopoietic support. FASEB J. 2020;34 (4):5435-5452

24. Morhayim J, van de Peppel J, Braakman E, et al. Osteoblasts secrete miRNA-containing extracellular vesicles that enhance expansion of human umbilical cord blood cells. Sci Rep. 2016;6:32034.

25. Renström J, Istvanffy R, Gauthier K, et al. Secreted frizzled-related protein 1 extrinsically regulates cycling activity and maintenance of hematopoietic stem cells. Cell Stem Cell. 2009;5(2):157-167.

26. Wilkinson $A C$, Ishida R, Kikuchi M, et al. Long-term ex vivo haematopoietic-stemcell expansion allows nonconditioned transplantation. Nature. 2019;571(7763): 117-121.

27. Zandstra PW, Petzer AL, Eaves CJ, Piret JM. Cellular determinants affecting the rate of cytokine in cultures of human hematopoietic cells. Biotechnol Bioeng. 1997;54(1):5866.

28. Yagi M, Ritchie KA, Sitnicka E, Storey C, Roth GJ, Bartelmez S. Sustained ex vivo expansion of hematopoietic stem cells mediated by thrombopoietin. Proc Natl Acad Sci U S A. 1999;96(14):8126-8131.

29. Ieyasu A, Ishida R, Kimura T, et al. An allrecombinant protein-based culture system specifically identifies hematopoietic stem cell maintenance factors. Stem Cell Rep. 2017;8(3):500-508

30. Oostendorp RA, Gilfillan S, Parmar A, et al. Oncostatin M-mediated regulation of KITligand-induced extracellular signal-regulated kinase signaling maintains hematopoietic repopulating activity of LinCD34+CD133+ cord blood cells. Stem Cells. 2008;26(8):2164-2172.

31. Boitano AE, Wang J, Romeo R, et al. Aryl hydrocarbon receptor antagonists promote the expansion of human hematopoietic stem cells. Science. 2010;329(5997):13451348

32. Fares I, Chagraoui J, Gareau Y, et al. Cord blood expansion. Pyrimidoindole derivatives are agonists of human hematopoietic stem cell self-renewal. Science. 2014;345 (6203):1509-1512.

33. Fares I, Rivest-Khan L, Cohen S, Sauvageau G. Small molecule regulation of normal and leukemic stem cells. Curr Opin Hematol. 2015;22(4):309-316.

34. Csaszar E, Kirouac DC, Yu M, et al. Rapid expansion of human hematopoietic stem cells by automated control of inhibitory feedback signaling. Cell Stem Cell. 2012;10 (2):218-229 